\title{
THE IMPACT OF PRIOR PROSTATIC SURGERY ON URINARY CONTINENCE IN PATIENTS UNDERGOING ORTHOTOPIC ILEAL NEOBLADDER
}

\author{
HOMERO O. DE ARRUDA, RUBENS SUAREZ, MIGUEL SROUGI, ADRIANO A. DE PAULA, \\ JOSÉ CURY
}

Division of Urology, Paulista School of Medicine, Federal University of Sao Paulo, UNIFESP, São Paulo, SP, Brazil

\begin{abstract}
Objective: To establish if previous surgery for benign prostatic hyperplasia (transurethral resection of the prostate or open prostatectomy), age, and preservation of prostatic apex can influence postoperative urinary continence in patients submitted to radical cystectomy and orthotopic ileal neobladder.

Patients and Methods: We analyzed 62 patients with bladder cancer who were treated with radical cystectomy and orthotopic ileal neobladder between 1987 and 1998 and had been followed for at least 24 months. The average age and median follow up were 61 years and 53 months, respectively. Postoperative urinary continence was correlated with 3 factors: patient age, preservation of prostatic apex during surgical excision and prior prostatic surgery for benign disease. Patients were defined as incontinent when they had to use more than 1 protective pad at the daytime.

Results: The overall incidence of urinary incontinence was $12.9 \%$ ( 8 out of 62 patients). The only statistically significant factor that impacted upon urinary continence was previous prostatic surgery, with respectively $33 \%$ versus $7 \%$ rate of incontinence for patients previously operated on and for those without previous operation ( $p=0.023$ odds ratio $=6.5,95 \%$ confidence interval). Preservation of prostatic apex did not reach difference, $12 \%$ versus $13 \%$, for those with and without preservation, and age also did not influence the postoperative continence rate.

Conclusions: Prior prostatic surgery for benign prostatic hyperplasia probably can increases the risk for postcystectomy incontinence and preservation of prostate apex did not affect the continence rate. This issue deserves to be considered by the surgeon and must be discussed previously with the patients when planning an orthotopic bladder replacement.
\end{abstract}

Key words: bladder; bladder neoplasms; urinary diversion; urinary reservoirs; continence Int Braz J Urol. 2003; 29: 502-506

\section{INTRODUCTION}

Radical cystectomy remains the golden standard for the treatment of invasive bladder cancer. In the last 10 to 15 years, significant surgical advances have been reached for urinary diversion following radical cystectomy, leading to an increased number of patients that have been subjected to an orthotopic ileal neobladder. As the result of improved clinical staging, earlier patient acceptance for radical surgery and improved postoperative support, there has been a significant decrease in the morbidity and mortality of these cases $(1,2)$

According to the pioneer work of Le Duc \& Camey (2), the principle of bladder replacement with ileum and the preservation of the distal urethral 
sphincter allowing urinary continence have shown to be technically feasible with an acceptable level of morbidity (3). Also, the principle of bowel detubularization used in the ileal Kock pouch (4) led to an improvement in the capacity/pressure ratio of the ileal reservoirs, making the technique more efficient and popularizing its use. A great number of different techniques for bladder substitution have been proposed in the last years, using the same basic principles: a high capacity and compliant reservoir with low pressure to preserve the integrity of the upper urinary tract and reconstruction that avoids ureteral reflux or obstruction. This advance promoted better urinary continence and more physiological micturition improving the patient's quality of life (5), which are crucially important for surgery acceptance in this patient subset.

In the present work, we studied daytime urinary continence in patients with orthotopic ileal neobladder and its relationship to age, prostatic apex preservation, and prior surgery for benign prostatic hyperplasia.

\section{PATIENTS AND METHODS}

A retrospective study of the charts from 62 patients with bladder cancer treated at the Federal University of São Paulo Medical School between 1987 and 1998 was performed. Only patients who were alive and followed for at least 24 months were included. The patients' age and postoperative follow up period ranged from 44 to 78 years $($ median $=61$ years) and from 24 to 122 months (median $=53$ months) respectively.

All patients had clinical stage T2 - T3 disease or irressectable superficial bladder carcinoma and underwent radical cystectomy and orthotopic ileal neobladder using $40-45 \mathrm{~cm}$ of detubularized ileum with a "J" configuration. The same surgeon using the same technique (6) performed all surgical procedures. In the male group, 38 patients were submitted to classical cystoprostatectomy with the anastomosis of the distal urethra to the most pendent portion of the ileal neobladder. In 24 patients, the prostatic apex was preserved, with removal of the proximal $2 / 3$ of the prostate gland. In the latter group the preserved distal prostate gland was anastomosed to the ileal reservoir and in all male patients, efforts were made to spare the cavernous neurovascular bundles. Postoperative staging revealed 5 patients $\mathrm{p}$ T0 N0, 11 patients $\mathrm{pTa}$ pT1 N0 M0, 38 patients pT2 - pT3 N0 M0 and 8 patients pT2 - pT3 N1 M0.

Three risk factors for urinary incontinence following surgery were evaluated, including patient's age, preservation of the prostatic apex at surgery and prior prostatic surgery (transurethral resection or open prostatectomy) for benign hyperplasia. Urinary incontinence was defined as the need for more than 1 protective pad per day during daytime after at least 2 years following operation (3).

\section{RESULTS}

Eight out of 62 patients (12.9\%) had persistent diurnal urinary incontinence after 2 years. The correlation between the possible risk factors and urinary incontinence is shown in the Table-1. All the values were submitted to a Fischer's exact test.

When considering patients' age, those who were 70 years old or more had a higher incidence of urinary incontinence compared to younger patients (18\% versus $10 \%$ ) but this difference did not reach statistic significance $(\mathrm{p}=0.604$ and odds ratio - OR $=0.52$ ). Preservation of the prostatic apex showed similar results with $3 / 24(12 \%)$ and $5 / 38$ (13\%) of

Table 1 - Risk factors and the incidence of daytime urinary incontinence. * More than one pad at daytime.

\begin{tabular}{|c|c|c|c|}
\hline & $\mathbf{N}$ & Incontinence $*(\%)$ & $\mathbf{p}$ \\
\hline Age & & $* *$ & 0.604 \\
\hline$<70$ & 53 & $6(11)$ & \\
\hline$>70$ & 9 & $2(20)$ & \\
\hline $\begin{array}{l}\text { Prostate apex } \\
\text { preservation }\end{array}$ & & & 1.000 \\
\hline Yes & 24 & $3(12)$ & \\
\hline No & 38 & $5(13)$ & \\
\hline $\begin{array}{l}\text { Previous prostate } \\
\text { surgery }\end{array}$ & & & 0.023 \\
\hline Yes & 15 & $5(33)$ & \\
\hline No & 42 & $3 \quad(7)$ & \\
\hline
\end{tabular}


the incontinence rate $(\mathrm{OR}=0.94, \mathrm{p}=1)$. From the 3 risk factors studied, the unique significant predictor for urinary incontinence was previous prostatic surgery. Previous prostate surgery was possible to be checked in only 57 patients, with transurethral prostate resection performed in 48 and open prostatectomy in 9. This group demonstrated in the outcome a $33 \%$ urinary incontinence rate versus $7 \%$ for the nonoperated group $(\mathrm{p}=0.023$ and $\mathrm{OR}=6.5$ with confidence interval $-\mathrm{CI}=95 \%)$.

\section{COMMENTS}

In the present study $54 / 62(79.1 \%)$ of the patients have diurnal continence according to our criteria and these figures were comparable to other published series where postoperative urinary continence ranged from $60 \%$ to $96 \%(1,3,5,7-9)$. In order to maintain erectile function and urinary continence, Schlling \& Friesen (10) since 1987, had already been made routine diagnostic staging of the cancer with transurethral resection of the prostate to permit a transprostatic selective cystectomy with an ileal bladder, cutting through the apex of the prostate thus, leaving a wide funnel-shaped tunnel of the prostatic urethra, for the anastomosis with the M or W shaped ileal reservoir. They achieve after a training period of 3 months, a frequency of miction of 3-4 times during the day and once during the night and all patients continent day and night. Park \& Montie (6), in 1998, studying the possible causes for incontinence following cystectomy and orthotopic neobladder noticed loss of primary failure-to-empty problem in $1 / 3$ of the cases due to low pouch compliance or high-pressure bowel contraction waves. Failure-to-store as a result of either urethral sphincter dysfunction secondary to surgical damage and inadequate length of the proximal urethra or compliance of the reservoir were found in the remaining 2/3 of the cases. In 1999, Hautmann (3) studied the recovery of urinary continence following radical cystectomy in 363 patients who had urinary reconstruction through an ileal neobladder and were followed for a median of 57 months (range 10 to 137). The rate of incontinence decreased over time from $8 \%$ to $4 \%$ up to 5 years, the point when there was no further improvement in urinary continence.
Gasparini et al. (11) studied continence after radical cystoprostatectomy and compared with patients continent after radical prostatectomy. In both groups, surgical techniques were modified to optimize preservation of the periurethral tissue and the prostatic apex. For the ileal neobladder group, 90\% were completely continent. The urethral sphincter mechanism was well preserved in these patients, with no significant difference between the 2 groups, neither in functional urethral length or in maximal urethral closure pressure. They concluded that continence is dependent upon an intact urethral sphincter mechanism as well as compliant and lowpressure reservoir; preserving as much of the distal urethral sphincter as possible may minimize either bladder or bladder substitute and incontinence. With the same objective and to determine the impact of preserving the prostatic apex on continence in these patients, Koraitim et al. (5) concluded that this preservation did not improve urinary continence and even, may present an element obstructing the evacuation of ileal neobladders. Recently, to minimize the risk of incontinence some authors (1115) suggest cystectomy with prostate sparing, although careful selection of the patients is mandatory to exclude occult prostate cancer. With this strategy, 90 - 97\% are fully continent (no pad) during the day and $95 \%$ void 1 to 2 times at night to stay dry (14).

Certainly, preserving the prostatic apex is easier to do and in accordance to the literature, we were expecting less incontinence, because the dissection beyond the sphincter could preserve the anatomic and functional integrity. Nonetheless, our study suggests that preserving the prostatic apex only, aiming to spare the distal urinary sphincter, did not decrease urinary incontinence rate. Incontinence varies from $13 \%$ to $12 \%$ respectively, for those without and with preservation of the apex, in the orthotopic neobladders. These findings has obvious clinical implications, since preservation of the distal part of the prostate in some cases can increase local bleeding during operation and may eventually interfere with neobladder emptying in the future (5). In case the results are really similar, there are more reasons to remove the prostate completely. 
Surprisingly, when comparing the results of continence between patients with and without previous prostate surgery, there was a high proportion on continents (93\%) in those who were not operated (39/42), in contrast to the $66 \%$ of the previous operated group (10/15). Unfortunately, it was only possible to check that from the 57 patients, 48 were submitted to a transurethral resection and 9 to an open simple prostatectomy. The real mechanisms that explain the higher incidence of incontinence in patients with prior history of prostate surgery undergoing radical cystectomy and orthotopic ileal neobladder were not determined in our study, but we can conceive 3 possible factors: 1) direct damage of the external sphincter during prostatic surgery, 2) fibrosis of the membranous urethra secondary to the surgical healing process or chronic prostatitis, 3) chronic external sphincter dysfunction secondary to its increased work following internal sphincter loss imposed by the previous prostatic surgery. Probably, as $85 \%$ of the patients had had transurethral resection it is easier to suppose that an intrinsic problem of the urethra at the sphincter level might have previously occurred. Furthermore, other studies on this issue and the neuromuscular participation of the prostatic apex in the mechanism of continence are necessary to clear the real cause of the problem.

In conclusion, according to our findings, we can theorize that patients submitted to prostatic surgery may be predisposed to urinary incontinence after orthotopic bladder replacement. Reviewing the literature, we did not find previous studies evaluating the role of prostatic surgery as a major risk factor for urinary incontinence in such patients. Even though we do not have the correct answers, it makes sense to take into account the need to preserve the prostatic apex during radical cystectomy with the scope of improving urinary continence as recently proposed (15). Finally, similarly to what happens with patients submitted to radical prostatectomy for prostate cancer (16), age had no significant influence on urinary continence outcome following cystoprostatectomy, although with such small values, the chi-square $p$ value is not accurate and we would need more patients to define it precisely.
Urologist and patients must acknowledge whatever adverse mechanism plays a role in these patients, the fact that past history of prostatic surgery for benign disease can increase the risk of urinary incontinence. This subset of patients must be advised when cystectomy and an orthotopic ileal neobladder are being considered for the treatment of invasive bladder cancer. Further prospective and randomized studies with separated groups correlating previous prostates surgery to a higher risk of urinary incontinence should be performed, in order to confirm our findings and lead to more appropriate care to patients with invasive bladder cancer.

\section{REFERENCES}

1. Bachor R, Frohneberg G, Miller K, Egghart G, Hautmann R: Continence after total bladder replacement: urodynamic analysis of the ileal neobladder. Br J Urol. 1990; 65: 462-6.

2. Le Duc A, Camey M: A procedure for avoiding reflux in uretero-ileal implantation during enterocystoplasty. J Urol. 1979; 85: 449-54.

3. Hautmann RE, de Petriconi R, Gottfried HW, Kleinschmidt K, Mattes R, Paiss T: The ileal neobladder: complications and functional results in 363 patients after 11 years of follow-up. J Urol. 1999; 161: 422-7.

4. Kock NG, Nilson AE, Nilsson LO, Norlen LJ, Philipson BM: Urinary diversion via a continent ileal reservoir: clinical results in 12 patients. J Urol. 1982; 128: 46975.

5. Koraitim MM, Atta MA, Foda MK: Impact of the prostatic apex on continence and urinary flow in patients with intestinal neobladder. Br J Urol. 1996; 78: 534-6.

6. Park JM, Montie JE: Mechanisms of incontinence and retention after orthotopic neobladder diversion. Urology. 1998; 51: 601-9.

7. Rogers E, Scardino PT: A simple ileal substitute bladder after radical cystectomy: experience with a modification of the Studer pouch. J Urol. 1995; 153: 1432-8.

8. Santucci RA, Park CH, Mayo ME, Lange PH: Continence and urodynamic parameters of continent urinary reservoirs: comparison of gastric, ileal, ileocolic, right colon and sigmoid segments. Urology. 1999; 54: 252-7. 
9. Studer EU, Zingg EJ: Ileal orthotopic bladder substitutes: what we have learned from 12 years' experience with 200 patients? Urol Clin North Am. 1997; 24: 781-93.

10. Schilling A. Friesen A: Transprostatic selective cystectomy with an ileal bladder. Eur Urol. 1990; 18: 253-7.

11. Gasparini ME, Hinman F Jr, Prest JC Jr, Schimdt RA Carroll PR: Continence after radical prostatectomy and total bladder replacement: a urodynamic analysis. J Urol. 1992; 148: 1861-4.

12. Vallancien G, Fettouh HAE, Cathelineal X, Baumert $\mathrm{H}$, Fromont G, Guillonneal B: Cystectomy with prostate sparing for bladder cancer in 100 patients: 10-year experience. J Urol. 2002; 168: 2413-17.

13. Colombo R, Bertini R, Salonia A, Da Pozzo LF,

Correspondence address:

Dr. Homero Oliveira de Arruda

Rua Napoleão de Barros, 715 / 2o.andar

São Paulo, SP, 04024-002, Brazil

Fax: + $55114521-9658$

E-mail: arrudas@dglnet.com.br

\section{EDITORIAL COMMENT}

This really is an interesting paper. It has a real message that, in the 42 men in whom they did an orthotopic ileal neobladder who had not had previous prostate surgery, 92 percent had daytime continence, while in the 15 patients who had had previous prostate surgery, this continence rate dropped to 66 percent. Since they were the same surgeons and since the continence rate for the people not having previous prostate surgery is the same as reported by other large studies, one has to feel that this difference is real and is related to the previous surgery.

Dr. Ralph W. de Vere White Professor and Chair, Department of Urology Director, Davis Cancer Center University of California Sacramento, California, USA
Montosi F, Brausi M, et al.: Nerve and seminal sparing radical cystectomy with orthotopic urinary diversion for select patients with superficial bladder cancer: an innovative surgical approach. J Urol. 2001; 165: 515.

14. Elbadawi A, Matheus R, Light JK, Wheeler TM. Immunohistochemical and ultrstructural study of rhabdosphincter component of the prostatic capsule. J Urol. 1997; 158: 1819-28.

15. Srougi M, Dall' Oglio M, Nesrallah LJ, Arruda HO, Ortiz V: Radical cystectomy with preservation of sexual function and urinary continence: descriptio of a new technique. Int Braz J Urol. 2003; 29: 336-44.

16. Steiner MS, Morton RA, Walsh PC: Impact of radical prostatectomy on urinary continence. J Urol. 1991; 145: 512-5.

Received: June 26, 2003 Accepted after revision: September 30, 2003

\section{EDITORIAL COMMENT}

The manuscript explores several factors that may affect urinary continence after orthotopic bladder construction. The authors used a strict but reasonable definition of incontinence; namely the need for greater than one pad a day. They report a previously unrecognized, or at least unreported, risk factor for incontinence after orthotopic bladder construction; namely a history of prior prostatic surgery. Then they proposed several mechanisms to explain this finding. Certainly this is information easily ascertained and elicited by a review of medical history, and it also may affect a number of patients facing cystectomy. However, the increase frequency of medical therapy for lower urinary tract symptoms will likely reduce the pool.

Dr. Paul F. Schellhammer Professor and Chair, Department of Urology Eastern Virginia Medical School Norfolk, Virginia, USA 